\title{
Heavy baryons in holographic QCD with higher dimensional degrees of freedom
}

\author{
Daisuke Fujii@* and Atsushi Hosaka๑ $\odot^{\dagger, \$}$ \\ Research Center for Nuclear Physics(RCNP), Osaka University, Ibaraki 567-0048, Japan
}

(Received 5 April 2020; accepted 20 May 2020; published 12 June 2020)

\begin{abstract}
We try to introduce heavy flavors to Sakai-Sugimoto model by regarding higher dimensional components of gauge fields as heavy mesons. Using the Forgács and Manton approach, we obtain a theory composed of heavy mesons and the instanton of light flavors. Applying the collective coordinate quantization method, we derived a mass formula of heavy baryons. In the leading order of $1 / m_{H}$ expansion ( $m_{H}$ the mass of a heavy quark), we find singlet and doublet states in the heavy quark symmetry (HQS). Also, we obtain the degenerate Roper like and odd parity excitations. By virtue of heavy meson degrees of freedom, our mass formula reproduces the mass ordering of $\Sigma_{c}^{*}$ and $\Lambda_{c}^{*}$ correctly.
\end{abstract}

DOI: $10.1103 /$ PhysRevD.101.126008

\section{INTRODUCTION}

In the past decades, hadron physics has experienced many new findings of exotic phenomena mostly containing charm and bottom (heavy) quarks that are not easily explained by conventional approaches, which is telling that we need new ideas [1,2], and references there in [3-6]. Due to the nonperturbative nature of the strong interaction dynamics, there still remains the problem of a missing link from quarks to hadrons. Holographic view of QCD has attracted much attention as one of guiding principle to fill that link. Among several alternatives, Sakai and Sugimoto proposed a D4-D8 brane construction that has lead to low energy effective actions for hadrons, where the model reproduces important features of spontaneous breaking of chiral symmetry including hadron resonances such as $\rho$ and $a_{1}$ mesons with a few parameters $[1,2]$.

The Sakai-Sugimoto model is a nine-dimensional gauge theory of flavor $\mathrm{SU}(2)$ on the D8-brane in the D4-brane background. Our model is an $\mathrm{SU}(2+1)$ extension of the Sakai-Sugimoto model. The gauge fields are denoted as $A_{M}^{a}$ where $a=1, \ldots, 8$ are for the flavor index and $M=$ $0, \ldots, 3, z, 6, \ldots, 9$ for gauge field components. In comparison with actual QCD, Sakai and Sugimoto have utilized five components, $A_{M=0, \ldots, 3, z}^{a}$, while the other four were

\footnotetext{
*daisuke@rcnp.osaka-u.ac.jp

†hosaka@rcnp.osaka-u.ac.jp

*Advanced Science Research Center, Japan Atomic Energy Agency, Tokai, Ibaraki 319-1195 Japan.

Published by the American Physical Society under the terms of the Creative Commons Attribution 4.0 International license. Further distribution of this work must maintain attribution to the author(s) and the published article's title, journal citation, and DOI. Funded by SCOAP ${ }^{3}$.
}

ignored when they derived a five-dimensional gauge theory [1]. Then the fifth-dimensional degrees of freedom play a role of generating various hadron resonances of light flavors of $u, d$ quarks in the four-dimensional space-time.

In this paper, using the extra-dimensional degrees of freedom, we attempt to construct a model with heavy flavors for the study of heavy baryons. The gauge field living in the extra higher dimensional space-time is transformed into heavy mesons by the method of dimensional reduction by Forgács and Manton [7,8]. The method leads to the field components that correspond to heavy mesons and light flavor instantons. Supplemented by a mass term, we propose a set of actions for heavy hadrons.

Once we establish the model in five-dimensional spacetime, we apply the standard method to the quantization of instantons for heavy baryons, from which we compute various physical quantities. We estimate masses of heavy baryons that are compared with existing experimental data. We would then like to study whether such a construction provides a reasonable description for not only conventional but also exotic states such as $P_{c}$ states.

This paper is organized as follows. In Sec. II, we outline our method of dimensional reduction applied to the SakaiSugimoto model with extra-dimensional components of the gauge fields identified with heavy mesons. In Sec. III, we apply the semiclassical method of collective coordinates to obtain physical baryons. Results are compared with experimental data.

\section{ACTION}

The $\mathrm{SU}(2+1)$ (light + heavy flavors) gauge fields on the probed D8 brane in the D4 brane background $R^{4} \times S^{5}$ have nine components, $A_{M}(M=0-3, U, \alpha)$, where $U$ is a radial coordinate of $S^{5}$ and $\alpha=\psi, \varphi, \theta_{1}, \theta_{2}$ angular 
coordinates of $S^{5}$. The gauge fields have also flavor components denoted by the index $a$, where $A_{M}=A_{M}^{a} \lambda^{a} / 2$ and $\lambda^{a}$ are the Gell-Mann matrices. In Refs. [1,2], the gauge field components on $S^{4}, A_{\alpha}$, were ignored. In the present work, by regarding $A_{\alpha}^{4-7}$ among $A_{\alpha}^{a}$ as heavy mesons, we try to introduce heavy flavors in the Sakai-Sugimoto (SS) model. When one reduces the dimensions of $S^{4}$, we will see that the vector fields $A_{\alpha}^{4-7}$ are transformed into scalar heavy meson fields. We should remark that in embedding the light and heavy mesons in the flavor $\mathrm{SU}(3)$ parametrization it is assumed that the SU(3) symmetry is not badly broken for the interaction among them and only their mass difference is the major source of the $\mathrm{SU}(3)$ breaking. The applicability of such an approach was discussed carefully in Ref. [9] in the bound state approach for hyperons. Our approach here is also based on the same argument.

Since there are two types of terms in our action, this section is divided into two subsections. First, in Sec. II A we explain how to reduce the dimensions of the higher dimensional Yang-Mills gauge theory. Second, in Sec. II B, we discuss the Chern-Simons term that we will need.

\section{A. The Yang-Mills part}

In our model, we treat $A_{\psi}$ and $A_{\varphi}$ components as heavy meson fields, and try to discuss a system of heavy mesons and nucleons. $A_{\theta_{1}}$ and $A_{\theta_{2}}$ components are ignored for the minimal use of the extra-dimensional degrees of freedom. The nucleons are described by instantons of $A_{1-3, z}$ components [10-12]. To reduce the nine-dimensional theory into a five-dimensional theory, we employ the method proposed by Forgács and Manton [7].

The Yang-Mills (YM) action that we use is given by the leading term of the DBI action of the SS model [1],

$$
\begin{aligned}
S_{D 8}^{\mathrm{DBI}} \simeq & T_{8}\left(2 \pi^{2} \alpha^{\prime}\right)^{2} \int d^{9} x e^{-\phi} \sqrt{-\operatorname{det} g} g^{M N} g^{P Q} \\
& \times \operatorname{tr}\left(\frac{1}{4} F_{M P}^{\mathrm{SU}(3)} F_{N Q}^{\mathrm{SU}(3)}\right)
\end{aligned}
$$

where $T_{8}=(2 \pi)^{-8} l_{s}^{-9}$ is the tension of the D8-brane, $\alpha^{\prime}=l_{s}^{2}, l_{s}$ the string length, and $\phi$ the dilaton field. $F_{M N}^{\mathrm{SU}(3)}$ is the field strength of flavor $\mathrm{SU}(3)$ gauge fields, and $F_{M N}^{\mathrm{SU}(3)}=\partial_{M} A_{N}-\partial_{N} A_{M}+i\left[A_{M}, A_{N}\right]$. Using the Minkowski metric, $\operatorname{diag}\left(\eta^{\mu \nu}\right)=(-1,+1,+1,+1)$, the metric of the D4 black brane is represented by the following $9 \times 9$ matrix:

$$
g^{M N}=\left(\begin{array}{ccc}
\left(\frac{R}{U}\right)^{3 / 2} \eta^{\mu \nu} & 0 & 0 \\
0 & \left(\frac{U}{R}\right)^{3 / 2} f & 0 \\
0 & 0 & \left(\frac{U}{R}\right)^{3 / 2} U^{-2} g^{\alpha \beta}\left(\Omega_{4}\right)
\end{array}\right),
$$

where $U$ is the radial coordinate of $S^{5}, R$ and $U_{K K}$ characterize the structure of $S^{5}$ and $f=1-U_{K K}^{3} / U^{3}$.

Now, we assume that the gauge field has an $\mathrm{SO}(3)$ spherical symmetry in the $(U, \psi, \varphi)$ space. It means that under $\mathrm{SO}(3)$ rotational transformations a variation of the gauge (vector) field is absorbed into a gauge transformation. To see this point let us first introduce the generators of $\mathrm{SO}(3)$ transformations $\xi_{m}$ :

$$
\begin{aligned}
& \xi_{1}=\xi_{1}^{M} \partial_{M}=\cos \varphi \frac{\partial}{\partial \psi}-\cot \psi \sin \varphi \frac{\partial}{\partial \varphi} \\
& \xi_{2}=\xi_{2}^{M} \partial_{M}=-\sin \varphi \frac{\partial}{\partial \psi}-\cot \psi \cos \varphi \frac{\partial}{\partial \varphi} \\
& \xi_{3}=\xi_{3}^{M} \partial_{M}=\frac{\partial}{\partial \varphi}
\end{aligned}
$$

When one represents the $(U, \psi, \varphi)$ space as Cartesian coordinate, $\xi_{m}$ are generators that causes rotations around each axis. Applying them to the gauge (vector) field, an infinitesimal variation is computed by

$$
\delta_{\xi_{m}} A_{M}=\epsilon \mathcal{L}_{\xi_{m}} A_{M}
$$

where $\mathcal{L}_{\xi_{m}}$ denotes the Lie derivative associated with $\xi_{m}$, and $\epsilon$ is an infinitesimal parameter.

Naively, we consider a symmetric field configuration satisfies $\delta_{\xi_{m}} A_{M}=0$. However, in the case of gauge fields, it is possible to consider a weaker condition, by relating the variation $\delta_{\xi_{m}} A_{M}$ with a gauge transformation, $g=\mathrm{e}^{i \epsilon W_{m}}$ with $W_{m}$ being an su(3) Lie algebra corresponding to $\xi_{m}$,

$$
\delta_{W_{m}} A_{M}=\epsilon D_{M} W_{m}
$$

where $D_{M} \equiv \partial_{M}+i\left[A_{M},\right]$ is a covariant derivative. If an $\mathrm{SO}(3)$ infinitesimal transformation (4) equals an infinitesimal gauge transformation, i.e.,

$$
\delta_{\xi_{m}} A_{M}=\delta_{W_{m}} A_{M},
$$

we can make the right-hand side zero by a gauge transformation. Explicitly, this equation can be written as

$$
\left(\partial_{M} \xi_{m}^{N}\right) A_{N}+\xi_{m}^{N} \partial_{N} A_{M}=\partial_{M} W_{m}+i\left[A_{M}, W_{m}\right] .
$$

It is important that for the dimensional reduction, the $\mathrm{SO}(3)$ space-time symmetry is related to the flavor $\mathrm{SU}(3)$ gauge symmetry through (7) [7].

In order to perform the dimensional reduction for our purpose, we employ a set of ansatze for field configurations $[7,8]$,

$$
W_{m}=\left(\Phi_{3} \frac{\sin \varphi}{\sin \psi}, \Phi_{3} \frac{\cos \varphi}{\sin \psi}, 0\right),
$$




$$
\begin{aligned}
A_{\mu, U} & =A_{\mu, U}\left(x^{\nu}, U\right), \\
A_{\psi} & =-\Phi_{1}\left(x^{\mu}, U\right), \\
A_{\varphi} & =\Phi_{2}\left(x^{\mu}, U\right) \sin \psi-\Phi_{3} \cos \psi,
\end{aligned}
$$

where $\Phi_{1,2}$ are a function of $\left(x^{\mu}, U\right)$ and $\Phi_{3}$ a constant. Then the constraint (7) becomes

$$
\begin{aligned}
{\left[\Phi_{3}, \Phi_{1}\right] } & =-i \Phi_{2}, \\
{\left[\Phi_{3}, \Phi_{2}\right] } & =i \Phi_{1}, \\
{\left[\Phi_{3}, A_{\mu, U}\right] } & =0 .
\end{aligned}
$$

If we substitute (8) and (9) for (1), we can perform the integration of the higher dimensional manifold $S^{4}$, resulting in a five-dimensional action. The result for the YM part (1) becomes $[7,8]$ :

$$
\begin{aligned}
S_{Y M}= & \kappa \int d^{4} x d z \operatorname{tr}\left[-\frac{1}{2} K^{-1 / 3} F_{\mu \nu}^{2}-K F_{\mu z}^{2}\right. \\
& -\frac{4}{9}\left(D_{\mu} \Phi_{m}\right)^{2}-\frac{4}{9} K^{4 / 3}\left(D_{z} \Phi_{m}\right)^{2} \\
& \left.-\frac{16}{81} K^{1 / 3}\left(i \epsilon_{r s t} \Phi_{t}+\left[\Phi_{r}, \Phi_{s}\right]\right)^{2}\right],
\end{aligned}
$$

where $\kappa=N_{c} \lambda / 216 \pi^{3}=a N_{c} \lambda, N_{c}$ is a color number, and $\lambda$ the t'Hooft coupling constant. We use the change of variables between $U$ and $z$ by $U^{3} / U_{K K}^{3}=1+z^{2}=K$. $R$ and $U_{K K}$ are expressed by Kaluza-Klein mass $M_{K K}$ [2]. In the following we set $M_{K K}=1$. We can recover an $M_{K K}$ dependence by dimensional analysis when needed.

Now, let us provide a solution for the symmetry relations (7) or (10). A derivation is given in the Appendix in some detail. The results are,

$$
\begin{gathered}
A_{\mu, z}=A_{\mu, z}^{1} \frac{\lambda_{1}}{2}+A_{\mu, z}^{2} \frac{\lambda_{2}}{2}+A_{\mu, z}^{3} \frac{\lambda_{3}}{2}+A_{\mu, z}^{8} \frac{\lambda_{8}}{2}, \\
\Phi=\frac{1}{2}\left(\begin{array}{ccc}
0 & 0 & \phi_{1} \\
0 & 0 & \phi_{2} \\
0 & 0 & 0
\end{array}\right), \quad \tilde{\Phi}=\frac{1}{2}\left(\begin{array}{ccc}
0 & 0 & 0 \\
0 & 0 & 0 \\
\phi_{1}^{*} & \phi_{2}^{*} & 0
\end{array}\right),
\end{gathered}
$$

where $\Phi=\Phi_{1}+i \Phi_{2}, \tilde{\Phi}=\Phi_{1}-i \Phi_{2}$ and $\phi_{1,2}$ are complex scaler fields [8]. These expressions imply that the gauge fields $A_{\mu, z}$ correspond to light mesons and $\phi_{i}$ to heavy mesons. In fact, conceptually, this is in accordance with a brane picture where one heavy brane is separated from two light branes. The $\phi$ field correspond to the string connecting light and heavy brane, and hence is regarded as a heavy meson.

Substituting the light-heavy decomposed fields (12) and (13) for the Yang-Mills action (11), we find

$$
\begin{aligned}
S_{Y M}= & \kappa \int d^{4} x d z\left\{\operatorname{tr}\left[-\frac{1}{2} K^{-1 / 3} F_{\mu \nu}^{2}-K F_{\mu z}^{2}\right]\right. \\
& -\frac{4}{9}\left(D_{\mu} \phi\right)^{\dagger}\left(D_{\mu} \phi\right)-\frac{4}{9} K^{4 / 3}\left(D_{z} \phi\right)^{\dagger}\left(D_{z} \phi\right) \\
& \left.-\frac{16}{81} K^{1 / 3}\left(\frac{12}{9}-2 \phi^{\dagger} \phi+\left(\phi^{\dagger} \phi\right)^{2}\right)^{2}\right\} .
\end{aligned}
$$

where $\phi^{\dagger}=\left(\phi_{1}^{*}, \phi_{2}^{*}\right)$ is a two component isospinor [8]. $F_{\mu \nu, z}$ is the field strength of the $\mathrm{SU}(2) \times \mathrm{U}(1)$ gauge fields (12), and $D_{\mu, z}$ a covariant derivative.

\section{B. The Chern-Simons part}

It is known that the Wess-Zumino-Witten (WZW) term plays a characteristic role for heavy baryon dynamics, providing an attraction (repulsion) between a (anti) heavy meson and soliton background [9]. To introduce the relevant term, we follow the argument of Ref. [13] and start from the following Chern-Simons (CS) term[14],

$$
\begin{aligned}
S_{C S} & =\frac{N_{c}}{24 \pi^{2}} \int \operatorname{tr} \mathcal{F}^{3} \\
\operatorname{tr} \mathcal{F}^{3} & =\mathrm{d} \omega_{5}(\mathcal{A}) \\
& =\mathrm{d}\left[\operatorname{tr}\left(\mathcal{A} \mathcal{F}^{2}-\frac{i}{2} \mathcal{A}^{3} \mathcal{F}-\frac{1}{10} \mathcal{A}^{5}\right)\right],
\end{aligned}
$$

where $\mathcal{F}$ is the field strength of $\mathcal{A}$, and the 1 -form $\mathcal{A}$ is $\mathcal{A}=\mathcal{A}_{M} d x^{M}=A_{M} d x^{M}+\hat{A}_{M} d x^{M}(M=0,1,2,3, z, s)$. Here in (16), we have included the U(1) gauge field $\hat{A}_{M}$ that did not appear in (14) to stabilize the instanton solution at a finite size as discussed in Ref. [12,13]. Physically, it corresponds to the $\omega$ meson and has been known to play an important role in stabilizing the Skyrmion also [15].

The U(1) term of (15) decomposes

$$
\begin{aligned}
S_{C S}= & \frac{N_{c}}{24 \pi^{2}} \int \operatorname{tr} F^{3} \\
& +\frac{N_{c}}{24 \pi^{2}} \frac{1}{\sqrt{2 N_{f}}} \int\left[3 \hat{A} \operatorname{tr} F^{2}+\frac{1}{2} \hat{A} \hat{F}^{2}\right],
\end{aligned}
$$

where in the second term we have used the Stokes's theorem to reduce the six-dimensional integral to the five-dimensional one. If we choose $A_{z}=0$ gauge, omit massive modes, and integrate over $z$, the first term is

$$
\frac{N_{c}}{24 \pi^{2}} \int \operatorname{tr} F^{3} \simeq-\frac{i N_{c}}{240 \pi^{2}} \int \operatorname{tr}\left(U d U^{-1}\right)^{5},
$$

which is nothing but the WZW term [13].

If we use a hedgehog solution for baryons in Refs. [12,13], the chiral field $U$ has the following form: 


$$
\left.U\right|_{s=0}=\exp \left(\begin{array}{cc}
i H(\mathbf{x}) \hat{\mathbf{x}} \cdot \boldsymbol{\tau} / f_{\pi} & 0 \\
0 & 0
\end{array}\right)
$$

where $\hat{\mathbf{x}}$ is a unit vector, $\boldsymbol{\tau}$ a Pauli matrix and $f_{\pi}$ the decay constant of the pion. The choice of $s=0$ corresponds to the boundary of the six-dimensional manifold on which the WZW term is defined. Using the instanton solution, the function $H(\mathbf{x})$ is given as

$$
\int_{-\infty}^{+\infty} d z^{\prime} A_{z}^{c l}\left(\mathbf{x}, z^{\prime}\right)=H(\mathbf{x}) \hat{\mathbf{x}} \cdot \boldsymbol{\tau} .
$$

Since the WZW term is identically zero for flavor SU(2), this term (17) vanishes without heavy mesons corresponding to $\lambda_{4-7}$. Keeping these components $\varphi(\mathbf{x})$ we write as the chiral field:

$$
\left.U\right|_{s=0}=\exp \left(\begin{array}{cc}
i H(\mathbf{x}) \hat{\mathbf{x}} \cdot \boldsymbol{\tau} / f_{\pi} & \varphi(\mathbf{x}) / f_{H} \\
\varphi^{\dagger}(\mathbf{x}) / f_{H} & 0
\end{array}\right)
$$

where $f_{H}$ is the decay constant of heavy mesons. As we will discuss later, the function $\varphi(\mathbf{x})$ corresponds to the lowest eigenmode of the heavy meson fields $\phi$ when expanded in the fifth $z$-dimension.

Substituting (20) for (17) we find

$$
\begin{aligned}
- & i \frac{N_{c}}{240 \pi^{2}} \int \operatorname{tr}\left(U \mathrm{~d} U^{-1}\right)^{5} \\
= & \frac{i N_{c}}{f_{H}^{2}} \int d^{4} x B^{\mu}\left(\varphi^{\dagger} D_{\mu} \varphi-\left(D_{\mu} \varphi\right)^{\dagger} \varphi\right),
\end{aligned}
$$

where $B_{\mu}$ is the baryon number current by the soliton,

$B^{\mu}=\frac{\epsilon^{\mu \nu \alpha \beta}}{24 \pi^{2}} \operatorname{tr}\left[\left(U_{\pi} \partial_{\nu} U_{\pi}^{-1}\right)\left(U_{\pi} \partial_{\alpha} U_{\pi}^{-1}\right)\left(U_{\pi} \partial_{\beta} U_{\pi}^{-1}\right)\right]$,

with $U_{\pi}=\exp \left(i H(\mathbf{x}) \hat{\mathbf{x}} \cdot \boldsymbol{\tau} / f_{\pi}\right)[16]$.

\section{The model action}

To complete our program, we need to introduce a mass term in the action, which is not easily done in the holographic method of Sakai-Sugimoto. Supplementing a mass term our model action is

$$
S=S_{Y M}+S_{C S}-m^{2} K^{1 / 3} \phi^{\dagger} \phi
$$

where the function $K^{1 / 3}$ is introduced in accordance with (14) in consideration of the curved nature of the fifthdimension.

\section{CLASSICAL SOLUTIONS}

\section{A. The instanton solutions}

To discuss baryon properties, we follow the semiclassical method, that is first we find a time-independent classical solution and then quantize it by introducing slowly moving time-dependent variables. Because the direction $z$ is curved and the time component is coupled, in Ref. [12] they performed the $1 / \lambda$ expansion and obtained a solution for the gauge configuration order by order. In the leading order, the $\mathrm{SU}(2) \in \mathrm{SU}(3)$ part of the gauge field $A_{M}^{c l}(\mathbf{x}, z)$ and the $\mathrm{U}(1)$ part $\hat{A}_{M}^{c l}(\mathbf{x}, z)$ are obtained as,

$$
\begin{gathered}
A_{M}^{c l}(\mathbf{x}, z)=-i f(\xi) g \partial_{M} g^{-1}, \\
g(\mathbf{x}, z)=\frac{(z-Z)-i(\mathbf{x}-\mathbf{X}) \cdot \boldsymbol{\tau}}{\xi}, \\
\hat{A}_{M}^{c l}=0,
\end{gathered}
$$

where $M=1,2,3, z$, and

$$
\begin{aligned}
f(\xi) & =\xi^{2} /\left(\xi^{2}+\rho^{2}\right), \\
\xi & =\sqrt{(\mathbf{x}-\mathbf{X})^{2}+(z-Z)^{2}} .
\end{aligned}
$$

Here the parameters $(\mathbf{X}, Z)$ and $\rho$ are the collective coordinates for the position (center) and size of the instanton, respectively. In the next to leading order, the time-components of the $\mathrm{SU}(2)$ and $\mathrm{U}(1)$ gauge field are obtained as,

$$
\begin{gathered}
A_{0}^{c l}=0, \\
\hat{A}_{0}^{c l}=\frac{1}{8 \pi^{2} a} \frac{1}{\xi^{2}}\left[1-\frac{\rho^{4}}{\left(\xi^{2}+\rho^{2}\right)^{2}}\right] .
\end{gathered}
$$

\section{B. The solution of $\phi$}

In the present model, we have a heavy meson field $\phi(\mathbf{x}, z)$ that also posses a time-independent classical solution. To find it, we first employ a mode expansion [1] by a complete set $\left\{\psi_{n}(z)\right\}$,

$$
\phi(\mathbf{x}, z)=\sum_{n=0} \varphi_{n}(\mathbf{x}) \psi_{n}(z)
$$

where $\varphi_{n}$ are two component isospinors. We can choose an arbitrary complete set $\left\{\psi_{n}(z)\right\}$, and therefore, we choose the one to diagonalize the kinetic and mass terms in the four-dimensional space-time. Such a complete set $\left\{\psi_{n}(z)\right\}$ satisfies the following eigenvalue equation:

$$
-\partial_{z}\left(K^{4 / 3} \partial_{z} \psi_{n}(z)\right)+m^{2} K^{1 / 3} \psi_{n}(z)=\lambda_{n} \psi_{n}(z) .
$$

These eigenstates $\psi_{n}(z)$ correspond to various meson resonances with their eigenvalues regarded as their squared masses. If we consider only the lowest eigenmode, the quadratic terms in $\phi$ of (14) become 


$$
\kappa \int d^{4} x\left[-\partial_{\mu} \varphi^{\dagger}(\mathbf{x}) \partial^{\mu} \varphi(\mathbf{x})-m_{H}^{2} \varphi^{\dagger}(\mathbf{x}) \varphi(\mathbf{x})\right],
$$

where $M=0,1,2,3, z, m_{H}=\sqrt{\lambda_{0}}$, and we redefine $\psi=\psi_{0}, \varphi=2 / 3 \varphi_{0}$. The mass parameter $m$ is determined such that $m_{H}$ becomes the heavy meson mass $(D(1870), B(5279))$.

To proceed further, we apply again the $1 / \lambda$ expansion. For this purpose, first we rescale the fields as follows [12]:

$\tilde{x}^{0}=x^{0}, \quad \tilde{x}^{M}=\lambda^{1 / 2} x^{M}$,

$\tilde{\mathcal{A}}_{0}=\mathcal{A}_{0}, \quad \tilde{\mathcal{A}}_{M}=\lambda^{-1 / 2} \mathcal{A}_{M}, \quad \tilde{\varphi}=\lambda^{-1 / 2} \varphi$,

where $M=1,2,3, z$. In the following calculations, we omit the tilde for simplicity. Then, the action for $\varphi, S_{\varphi}$ becomes to the leading order of $1 / \lambda$ expansion

$$
S_{\varphi} \sim a N_{c} \lambda^{1} \int d^{4} x\left(-\partial_{i} \varphi^{\dagger} \partial^{i} \varphi-\varphi^{\dagger}\left(\int d z \psi^{2} \mathcal{A}_{M}^{2}\right) \varphi\right) .
$$

Using the solution (24), $\mathcal{A}_{M}^{2}$ is proportional to identity matrix. Therefore, to solve the equation of motion for $\varphi(\mathbf{x})$ we can decompose the two component $\mathrm{SU}(2)$ spinor $\varphi(\mathbf{x})$ into $f(\mathbf{x}) \chi$, where $\chi$ is a two component spinor. Then, the resulting equation of motion for $\varphi$ is given as

$$
\partial_{r}^{2} f+\frac{2}{r} \partial_{r} f-\left(3 \int d z \frac{\psi^{2}\left(z^{2}+r^{2}\right)}{\left(z^{2}+r^{2}+\rho^{2}\right)^{2}}\right) f=0 .
$$

To solve this equation, it is convenient to rescale the variable $\xi \rightarrow \rho \xi$. First, we should discuss the asymptotic behavior. At $r \rightarrow 0$, the third term of (33) approaches zero, so we note that the asymptotic behavior of $f$ is $f \sim r^{-1}$. Next, to see this at $r \rightarrow \infty$, we multiply (33) by $r^{2}$. Then, (33) becomes

$$
r^{2} \partial_{r}^{2} f+r \partial_{r} f-\left(3 \int d z \psi^{2} \frac{\left(z^{2} / r^{2}+1\right)}{\left(z^{2} / r^{2}+1+1 / r^{2}\right)^{2}}\right) f=0 .
$$

If $z$ is small, the integrand of the third term of (34) is

$$
\frac{\left(z^{2} / r^{2}+1\right)}{\left(z^{2} / r^{2}+1+1 / r^{2}\right)^{2}} \sim 1 .
$$

Also if $z$ is large, that term becomes smaller than 1 . However, in this case $\psi$ becomes almost zero, so in this region, the third term does not contribute to the equation of motion (34). Therefore, we can set the third term equals $3 f$. After all, $\left.f\right|_{r \rightarrow \infty}$ satisfy

$$
r^{2} \partial_{r}^{2} f+r \partial_{r} f-3 f=0 .
$$

Therefore, the asymptotic behavior at $r \rightarrow \infty$ is

$$
f \sim r \frac{-1-\sqrt{13}}{2} .
$$

We have solved Eq. (33) numerically satisfying the above asymptotic behaviors. This solution will be used when quantizing the classical solution and obtaining the mass formula for physical baryons.

\section{QUANTIZATION}

In Sec. III, we have solved the static classical solutions of an instanton and $\phi$. In the collective quantization method, we consider the dynamics of a soliton in a moduli space parametrized collective coordinates, and by regarding them as canonical variables.

\section{A. Collective coordinates}

In our model, collective coordinates are as follows [12]:

(i) Position of the instanton $(\mathbf{X}, Z)$

(ii) Size of the instanton $\rho$

(iii) $\mathrm{SU}(2)$ orientation $V$

(iv) Two component $\mathrm{SU}(2)$ spinor $\chi$ where $(\mathbf{X}, Z)$ and $\rho$ are the position and size of the instanton, respectively, and $V$ the $\mathrm{SU}(2)$ matrix corresponding to soliton rotations. In addition to these coordinates, we need the two component $\mathrm{SU}(2)$ spinor $\chi$ corresponding to the vibration of heavy mesons:

$$
\phi=f(\mathbf{x}) \psi(z)\left(\begin{array}{l}
\chi_{1} \\
\chi_{2}
\end{array}\right)=f(\mathbf{x}) \psi(z) \chi .
$$

These collective coordinates describe time-dependent collective motions of the classical solutions. Since the present theory is based on a gauge theory, we need to be a bit careful [17]. The collective coordinates introduce motions along the gauge orbits that cannot be physical motions. These unphysical motions can be removed by the following prescription [12],

$$
\begin{gathered}
A_{M}\left(t, x^{N}\right)=V A_{M}^{c l}\left(x^{N} ; X^{N}(t), \rho(t)\right) V^{-1}-i V \partial_{M} V^{-1}, \\
\phi\left(t, x^{N}\right)=V \phi^{c l}\left(x^{N} ; \rho(t), \chi(t)\right),
\end{gathered}
$$

where $V=V\left(t, x^{N}\right)$ is an element of the gauge group $\mathrm{SU}(2)$. In the $A_{0}=0$ gauge with imposing the Gauss's law:

$$
D_{M}^{c l}\left(\dot{X}^{N} \frac{\partial}{\partial X^{N}} A_{M}^{c l}+\dot{\rho} \frac{\partial}{\partial \rho} A_{M}^{c l}-D_{M}^{c l} \Phi\right)=0
$$

where $M, N=1,2,3, z, \Phi=-i V^{-1} \dot{V}$ and $D_{M}^{c l}=\partial_{M}+i\left[A_{M}^{c l},\right]$. By having the solution of $\Phi$ to (38) [12], spurious motions along the gauge orbits are removed in the collective motions of (36). 


\section{B. Heavy meson field}

The action for the heavy meson field $\varphi, S_{\varphi}$, is

$$
\begin{aligned}
S_{\varphi}= & a N_{c} \int d^{4} x\left[\lambda^{1}\left(-\partial_{i} \varphi^{\dagger} \partial^{i} \varphi-\varphi^{\dagger}\left(\int d z \psi^{2} \mathcal{A}_{M}^{2}\right) \varphi\right)\right. \\
& \left.+\lambda^{0}\left(\int d z \psi^{2}\left(D_{0} \varphi\right)^{\dagger} D_{0} \varphi-m_{H}^{2} \varphi^{\dagger} \varphi\right)\right] \\
& +\lambda^{0} \frac{i N_{c}}{f_{H}^{2}} \int d^{4} x B^{\mu}\left(\varphi^{\dagger} D_{\mu} \varphi-\left(D_{\mu} \varphi\right)^{\dagger} \varphi\right),
\end{aligned}
$$

where the covariant derivative $D_{0}$ is defined as $D_{0} \varphi=$ $\partial_{0} \varphi+i \mathcal{A}_{0} \varphi$.

It is convenient to introduce the heavy meson field as [18]

$$
\phi=e^{\mp i m_{H} t} \tilde{\boldsymbol{\phi}}=f(\mathbf{x}) \psi(z) e^{\mp i m_{H} t} \tilde{\chi}(t),
$$

where $-/+$ correspond to heavy/antiheavy mesons. Then, if we only consider the leading terms of $1 / m_{H}$ expansion and substitute the solutions (37) for (39), the first line of (39) is zero and the second line becomes

$$
\begin{aligned}
& \int d^{4} x d z f^{2} \psi^{2}\left[\left(D_{0}(V \chi)\right)^{\dagger} D_{0}(V \chi)-m_{H}^{2} \chi^{\dagger} \chi\right] \\
& \simeq 2 m_{H} \int d^{4} x d z f^{2} \psi^{2} \tilde{\chi}^{\dagger} D_{0} \tilde{\chi} .
\end{aligned}
$$

\section{Quantization}

By employing the normalization $a N_{c} \int d^{3} x d z f^{2} \psi^{2}=1$, absorbing the coefficient of the kinetic term of $\tilde{\chi}$ and integrating over the space of $\left(x^{\mu}, z\right)$, finally we obtain the action of collective motions as follows [12]:

$$
\begin{aligned}
& \int d t\left[L_{X}+L_{Z}+L_{y}\right]+\mathcal{O}\left(\lambda^{-1}, m_{H}^{-1}\right), \\
& L_{X}=-M_{0}+\frac{m_{X}}{2} \dot{\mathbf{X}}^{2}, \\
& L_{Z}=\frac{M_{Z}}{2} \dot{Z}^{2}-\frac{m_{Z} \omega_{Z}^{2}}{2} Z^{2}, \\
& L_{y}=\frac{m_{y}}{2} \dot{y}_{I}^{2}-\frac{m_{y} \omega_{\rho}^{2}}{2} \rho^{2}-\frac{Q}{\rho^{2}}, \\
& L_{\chi}= \pm i \tilde{\chi}^{\dagger} \partial_{t} \tilde{\chi} \pm A \frac{N_{c}}{\rho^{2}} \tilde{\chi}^{\dagger} \tilde{\chi},
\end{aligned}
$$

with

$$
\begin{aligned}
& M_{0}=8 \pi^{2} \kappa, \\
& m_{X}=m_{Z}=8 \pi^{2} a N_{c}, \quad m_{y}=16 \pi^{2} a N_{c}, \\
& \omega_{Z}^{2}=\frac{2}{3}, \quad \omega_{\rho}^{2}=\frac{1}{6}, \quad Q=\frac{N_{c}^{2}}{40 \pi^{2} a} .
\end{aligned}
$$

Also if we consider only the leading terms of $1 / m_{H}$ expansion, then only the time component contributes and therefore $B^{\mu}$ becomes

$$
B^{0}=\frac{1}{2 \pi^{2}} \frac{\sin ^{2} H}{r^{2}} \frac{d H}{d r} .
$$

Therefore, $A$ is written as

$A=\frac{4}{\pi f_{H}^{2}} \int d r \sin ^{2} H \frac{d H}{d r} f^{2}-a \int d^{3} x d z \hat{A}_{0}^{c l} \psi^{2} f^{2}$,

where using the rescale $x^{M} \rightarrow \rho x^{M}, \hat{A}_{0}^{c l}$ is

$$
\hat{A}_{0}^{c l}=\frac{1}{8 \pi^{2} a} \frac{1}{\xi^{2}}\left[1-\frac{1}{\left(\xi^{2}+1\right)^{2}}\right] .
$$

Therefore, we quantize the system with the canonical variables of these collective coordinates.

Before deriving the mass formula, we consider quantum numbers of the heavy mesons [9]. In the classical solution, the heavy meson fields have the spin 0 and the isospin $1 / 2$. First, we consider the isospin rotation. By the isospin rotation $g_{I}=e^{i \boldsymbol{\theta} \cdot \mathbf{I}}$, the gauge fields are transformed into

$$
\begin{aligned}
A_{M} & \rightarrow g_{I} A_{M} g_{I}^{-1}-i g_{I} \partial_{M} g_{I}^{-1} \\
& =\left(g_{I} V\right) A_{M}^{c l}\left(g_{I} V\right)^{-1}-i\left(g_{I} V\right) \partial_{M}\left(g_{I} V\right)^{-1} .
\end{aligned}
$$

On the other hand, the heavy meson field transforms

$$
V \tilde{\chi} \rightarrow g_{I} V \tilde{\chi}
$$

Therefore, $V$ carries the isospin, and $V$ and $\tilde{\chi}$ have the following transformation properties:

$$
\left\{\begin{array}{l}
V \rightarrow g_{I} V \\
\tilde{\chi} \rightarrow \tilde{\chi}
\end{array}\right.
$$

Second, we consider the spatial rotation. When the gauge transformation which is equivalent to the spatial rotation is written as $g_{J}=e^{i \theta \cdot \mathbf{J}}$, spatial rotation act the gauge field as follows:

$$
\begin{aligned}
A_{M}\left(t, R_{N P} x^{P}\right)= & V A_{M}^{c l}\left(R_{N P} x^{P} ; R_{N P} X^{P}\right) V^{-1}-i V V^{-1} \\
= & \left(V e^{-i \boldsymbol{\theta} \cdot \mathbf{I}}\right) A_{M}^{c l}\left(x^{N} ; X^{N}\right)\left(V e^{i \boldsymbol{\theta} \cdot \mathbf{I}}\right)^{-1} \\
& -i\left(V e^{-i \boldsymbol{\theta} \cdot \mathbf{I}}\right) \partial_{M}\left(V e^{i \boldsymbol{\theta} \cdot \mathbf{I}}\right)^{-1}
\end{aligned}
$$

where the hedgehog like structure relates the spatial rotation to isospin rotation, and so the spatial rotation is expressed by $g_{I}$. Also, the scalar field is transformed into

$$
\begin{aligned}
V \tilde{\phi}(t, x) \rightarrow V \tilde{\phi}\left(t, R_{M N} x^{N}\right) & =V e^{i \theta \cdot \mathbf{J} \tilde{\phi}} \\
& =V e^{-i \theta \cdot \mathbf{I}} f \psi e^{i \theta \cdot \mathbf{T}} \tilde{\chi}(t),
\end{aligned}
$$


where $\mathbf{T}=\mathbf{J}+\mathbf{I}$ is the grand spin operator. Therefore, $V$ and $\chi$ have the following transformation properties:

$$
\left\{\begin{array}{l}
V \rightarrow V e^{-i \boldsymbol{\theta} \cdot \mathbf{I}} \\
\tilde{\chi} \rightarrow e^{i \boldsymbol{\theta} \cdot \mathbf{T}} \tilde{\chi} .
\end{array}\right.
$$

From the above, after doing the collective rotation, $\tilde{\chi}$ has the spin $1 / 2$ and the isospin 0 . Thus, we should quantize $\tilde{\chi}$ as fermions:

$$
\left\{\tilde{\chi}_{i}, \tilde{\chi}_{j}^{\dagger}\right\}=\tilde{\chi}_{i} \tilde{\chi}_{j}^{\dagger}+\tilde{\chi}_{j}^{\dagger} \tilde{\chi}_{i}=\delta_{i j}
$$

\section{Mass formula}

By collecting the terms proportional to $1 / \rho^{2}$ in (42), our collective Hamiltonian takes essentially the same form as that of Ref. [12]. Therefore, we can follow the same quantization procedure, resulting in the mass formula as follows,

$$
\begin{aligned}
M= & M_{0}+\left(N_{Q}+N_{\bar{Q}}\right) m_{H} \\
& +\sqrt{\frac{(l+1)^{2}}{6}+\frac{2 N_{c}^{2}}{15}\left(1-\frac{40 a \pi^{2} A}{N_{c}}\left(N_{Q}-N_{\bar{Q}}\right)\right)} M_{K K} \\
& +\frac{2\left(n_{\rho}+n_{Z}\right)+2}{\sqrt{6}} M_{K K}
\end{aligned}
$$

where $M_{0}$ is the instanton mass, $N_{Q / \bar{Q}}$ the number of heavy/antiheavy mesons. We emphasize that our mass formula contains a numerical constant $A$ in the second line. This is a unique feature of our model construction. As we will see shortly, this term plays a crucial role in reproducing the mass ordering of $\Sigma_{c}$ and $\Lambda_{c}^{*}$ correctly as in experimental data.

We find that the spin $\mathbf{J}$ and the isospin $\mathbf{I}$ of the instanton are both $l / 2$. The spin of the baryons is the sum of spins of the instanton and heavy mesons. In the SS model, parity transformation is defined by $x^{M} \rightarrow-x^{M}$. Also, when $n_{Z}$ is even or odd, the wave function of $n_{Z}$ has parity even or odd. Therefore, the quantum numbers $\left(n_{\rho}, n_{z}\right)$ correspond to radial excitations and those which flip parity, respectively. Note that $\tilde{\phi}=f \psi \tilde{\chi}$ has parity even.

Parameters in or mass are $\left(M_{0}, M_{K K}, m, f_{\pi}\right)$ and are shown in Table I. As explained in Sec. III, $m$ is determined such that $m_{H}$ becomes the heavy meson mass $(D(1870), B(5279))$. Also, we use $f_{D} / f_{\pi}=1.7$ and $f_{B} / f_{\pi}=1.6$ as in Ref. [19], and the pion decay constant is $f_{\pi}=61.2 \mathrm{MeV}$ which is about $30 \%$ smaller than the experimental value 93.2 MeV [19]. For the decay constants of a heavy meson $f_{H}(H=D$ or $B)$, we have chosen it for the charmed and bottomed baryons, separately. For the Kalza-Klein mass, we use $M_{K K}=500 \mathrm{MeV}$ which is the same value as in Ref. [12]. Having these inputs, there is only one free parameter $M_{0}$ which is fixed to reproduced
TABLE I. Parameters in our model.

\begin{tabular}{ccccc}
\hline \hline$M_{0}(\mathrm{MeV})$ & $M_{K K}(\mathrm{MeV})$ & $m / M_{K K}$ & $m / M_{K K}$ & $f_{\pi} / M_{K K}$ \\
\hline-572 & 500 & $\begin{array}{c}4.385 \\
\text { (charm) }\end{array}$ & $\begin{array}{c}10.62 \\
\text { (bottom) }\end{array}$ & 0.122 \\
\hline \hline
\end{tabular}

the mass of $\Lambda_{c}$ (2286). We are then interested in mass differences of baryons. We note that our mass formula (54) differs from that of Ref. [20] in the term proportional to $A$. In our model, the term depends on the heavy meson decay constant $f_{H}$, while that of Ref. [20] does not have such parameter dependence. From (45) with the decay constant values as in Table I, we find $A=0.078$ for charm and $A=$ 3.7 for bottom sectors, respectively.

Results are summarized in Table II.

These results have some characteristic properties as follows.

(i) The quantum numbers $\left(n_{\rho}, n_{z}\right)$ physically correspond to the Roper and the odd parity excitations. As observed in the previous works [12], the mass formula (54) indicates the degeneracy between them which agrees well with experimental data for the light flavor sector, the feature that is difficult to be explained by a naive quark model. This feature seems to be generalized to strange baryons [21]. Whether this also extends to charm and bottom sectors is an interesting question. Possible candidates are $\Lambda_{c}(2765)$ and $\Lambda_{b}(6072)$, while their spin and parity are to be determined.

(ii) If we expand the mass formula (54) by $1 / N_{c}$, the mass splitting of $\Lambda_{c}$ and $\Sigma_{c}$ is proportional to $1 / N_{c}$. This splitting is related to the spin-spin interaction, and the $N_{c}$ dependency is consistent to that of the $1 / N_{c}$ expansion scheme.

(iii) Because we have included only the leading terms of $1 / m_{H}$ expansion, we have obtained the heavy quark symmetry (HQS) singlet $\Lambda_{c, b}\left(0 \frac{1}{2}^{+}\right)$and the degenerate doublet $\Sigma_{c, b}\left(1 \frac{1}{2}^{+}, 1 \frac{3}{2}^{+}\right)$. On the other hand, the lowest $\Lambda_{c, b}\left(0 \frac{1}{2}-\right)$ and $\Lambda_{c, b}\left(0 \frac{3}{2}^{-}\right)$in a quark model do not exist in the present model, because this state is considered to correspond to the $\lambda$ mode. This is the reason that we put the mass value 2595 in parentheses in Table II. In our mass formula the excited states of baryons are described by excitations of the instanton, which correspond to $\rho$ modes in a quark model language.

(iv) Empirically, the mass splitting of $\Lambda_{c}$ and $\Lambda_{c}^{*}$ is about twice larger than that of $\Lambda_{c}$ and $\Sigma_{c}$. In the present study, the value of $A$ plays an important role to make this order of baryon masses. In particular, for $\Delta_{\Sigma_{c}-\Lambda_{c}} \equiv M\left(\Sigma_{c}\right)-M\left(\Lambda_{c}\right)$ and $\Delta_{\Lambda_{c}^{*}-\Lambda_{c}} \equiv M\left(\Lambda_{c}^{*}\right)-$ $M\left(\Lambda_{c}\right)$, we have $\Delta_{\Sigma_{c}-\Lambda_{c}}<\Delta_{\Lambda_{c}^{*}-\Lambda_{c}}$ in accordance with the experimental data, while the formula in [20] results in the reversed relation. Let $B$ be 
TABLE II. Predictions of our mass formula for the charmed and bottomed baryons in comparison with experimental data where available. Masses of heavy quark doublet, for instance $\Sigma_{c}$ and $\Sigma_{c}^{*}$, are degenerate in the heavy meson limit $m_{H} \rightarrow \infty$.

\begin{tabular}{|c|c|c|c|c|c|c|c|c|}
\hline$B$ & $I J^{P}$ & $l$ & $n_{\rho}$ & $n_{z}$ & $N_{Q}$ & $N_{\bar{Q}}$ & Our model/MeV & exp./MeV \\
\hline$\Lambda_{c}$ & $0 \frac{1}{2}{ }^{+}$ & 0 & 0 & 0 & 1 & 0 & [2286] & 2286 \\
\hline \multirow[t]{2}{*}{$\Sigma_{c}$} & $1 \frac{1}{2}+$ & 2 & 0 & 0 & 1 & 0 & 2523 & 2453 \\
\hline & $1 \frac{3}{2}^{+}$ & 2 & 0 & 0 & 1 & 0 & 2523 & 2520 \\
\hline \multirow[t]{2}{*}{$\Lambda_{c}^{*}$} & $0 \frac{1}{2}-$ & 0 & 0 & 1 & 1 & 0 & 2694 & $(2595)$ \\
\hline & $0 \frac{1}{2}^{+}$ & 0 & 1 & 0 & 1 & 0 & 2694 & $(2765)$ \\
\hline \multirow[t]{2}{*}{$\Sigma_{c}^{*}$} & $1 \frac{1}{2}^{-}, 1 \frac{3}{2}^{-}$ & 2 & 0 & 1 & 1 & 0 & 2931 & $\ldots$ \\
\hline & $1 \frac{1}{2}^{+}, 1 \frac{3}{2}^{+}$ & 2 & 1 & 0 & 1 & 0 & 2931 & $\ldots$ \\
\hline$P_{c}$ & $\frac{1}{2} \frac{1}{2},-\frac{13}{2}-$ & 1 & 0 & 0 & 1 & 1 & 4255 & $4312 / 4380 / 4440,4457$ \\
\hline \multirow[t]{2}{*}{$P_{c}^{*}$} & $\frac{1}{2} \frac{1}{2}^{-}, \frac{13}{2} \frac{-}{2}^{-}$ & 1 & 0 & 1 & 1 & 1 & 4664 & $\ldots$ \\
\hline & $\frac{1}{2} \frac{1}{2}^{+}, \frac{13}{2}{ }^{+}$ & 1 & 1 & 0 & 1 & 1 & 4663 & $\ldots$ \\
\hline$\Lambda_{b}$ & $0 \frac{1}{2}^{+}$ & 0 & 0 & 0 & 1 & 0 & 5676 & 5620 \\
\hline \multirow[t]{2}{*}{$\Sigma_{b}$} & $1 \frac{1}{2}+$ & 2 & 0 & 0 & 1 & 0 & 5919 & 5810 \\
\hline & $1 \frac{3}{2}^{+}$ & 2 & 0 & 0 & 1 & 0 & 5919 & 5830 \\
\hline \multirow[t]{2}{*}{$\Lambda_{b}^{*}$} & $0 \frac{1}{2}-$ & 0 & 0 & 1 & 1 & 0 & 6084 & 5912 \\
\hline & $0 \frac{1}{2}^{+}$ & 0 & 1 & 0 & 1 & 0 & 6084 & $(6072)$ \\
\hline \multirow[t]{2}{*}{$\Sigma_{b}^{*}$} & $1 \frac{1}{2}^{-}, 1 \frac{3}{2}^{-}$ & 2 & 0 & 1 & 1 & 0 & 6327 & $\ldots$ \\
\hline & $1 \frac{1}{2}^{+}, 1 \frac{3}{2}+$ & 2 & 1 & 0 & 1 & 0 & 6327 & $\ldots$ \\
\hline$P_{b}$ & $\frac{1}{2} \frac{1}{2}^{-}, \frac{13}{2} \frac{-}{2}^{-}$ & 1 & 0 & 0 & 1 & 1 & 11070 & $\ldots$ \\
\hline \multirow[t]{2}{*}{$P_{b}^{*}$} & $\frac{1}{2} \frac{1}{2}^{-}, \frac{13}{2} \frac{-}{2}^{-}$ & 1 & 0 & 1 & 1 & 1 & 11480 & $\ldots$ \\
\hline & $\frac{1}{2} \frac{1}{2}^{+}, \frac{1}{2} \frac{3}{2}^{+}$ & 1 & 1 & 0 & 1 & 1 & 11480 & $\ldots$ \\
\hline
\end{tabular}

$$
B=1-\frac{40 a \pi^{2} A}{N_{c}}
$$

For $B=0$, we find $\Delta_{\Sigma_{c}-\Lambda_{c}}=\Delta_{\Lambda_{c}^{*}-\Lambda_{c}}$. As $A$ becomes smaller (i.e., $B$ becomes larger), $\Delta_{\Sigma_{c}-\Lambda_{c}}$ becomes larger, and at some point, $\Delta_{\Lambda_{c}^{*}-\Lambda_{c}}$ equals $2 \Delta_{\Sigma_{c}-\Lambda_{c}}$.

(v) Our model has hidden charmed pentaquark states corresponding to $P_{c}(4312 / 4380 / 4440,4457)$ states reported recently $[22,23]$. Similarly, we predicted the mass of hidden bottomed pentaquark states not yet observed, which we denote by $P_{b}$ here. Since the soliton has the same value of the spin and the isospin which stems from the hedgehog structure, our mass formula cannot generate the $P_{c}$ state with the $\operatorname{spin} \frac{1}{2} \frac{5}{2}^{+}$.

\section{CONCLUSION}

In this paper, we aimed to apply the holographic model proposed by Sakai and Sugimoto to heavy flavor baryons, and derive a mass formula. In our model, extra-dimensional components of the gauge fields omitted in Ref. [1,2] have been interpreted as heavy mesons. The gauge fields living in $S^{4}$ have been transformed into heavy mesons by the Forgács-Manton method, and we have obtained the action composed of light and heavy mesons. Then, heavy baryons have represented as composite states of heavy mesons and an instanton composed by light flavors. In addition to the collective coordinates used in [12], our model has the dynamical variable corresponding to the vibration of heavy mesons. We have performed the collective coordinate quantization of the system consisted of these coordinates, and obtained the mass formula of heavy baryons. When quantizing our model, as in Ref. [9], heavy mesons behave as heavy quarks, which was referred to as transmutation of quantum numbers in the intrinsic frame of the hedgehog instanton [20]. The mass formula has given the mass spectra that are compared with existing experimental data.

In our model, we have considered the limit of the large $N_{c}$ and the t'Hooft coupling $\lambda$ as in [12], and also took the limit of large $m_{H}$. We have treated the only leading terms of $1 / m_{H}$, so we have obtained the HQS singlet $\Lambda_{c, b}\left(0 \frac{1}{2}^{+}\right)$and the doublet $\Sigma_{c, b}\left(1 \frac{1}{2}^{+}, 1 \frac{3}{2}^{+}\right)$. Also, our mass formula has yielded the degenerate Roper like and odd parity excitations. Moreover, we have realized the mass ordering $\Delta_{\Sigma_{c}-\Lambda_{c}}<\Delta_{\Lambda_{c}^{*}-\Lambda_{c}}$ in accordance with the experimental data. Furthermore, our model has hidden charmed pentaquark states $P_{c}(4312 / 4380 / 4440,4457)$ reported recently $[22,23]$. Similarly, we have predicted the masses of hidden bottomed pentaquark states not yet observed. 


\section{ACKNOWLEDGMENTS}

This work is supported in part by JSPS KAKENHI No. JP17K05441 (C) and Grants-in-Aid for Scientific Research on Innovative Areas (No. 18H05407).

\section{APPENDIX: SOLVING CONSTRAINTS}

In this Appendix, we will solve following constraints:

$$
\begin{gathered}
{\left[\Phi_{3}, \Phi\right]=-\Phi,} \\
{\left[\Phi_{3}, \tilde{\Phi}\right]=\tilde{\Phi},} \\
{\left[\Phi_{3}, A_{\mu, z}\right]=0,}
\end{gathered}
$$

where $\Phi=\Phi_{1}+i \Phi_{2}, \tilde{\Phi}=\Phi_{1}-i \Phi_{2}$. By using the Gellmann matrices $\lambda_{a}$, we can define the Cartan's standard form:

$$
\begin{aligned}
H_{1} & =\frac{\lambda_{3}}{2}, \quad H_{2}=\frac{\lambda_{8}}{2}, \\
E_{ \pm \gamma} & =\frac{1}{2}\left(\frac{\lambda_{1}}{2} \pm i \frac{\lambda_{2}}{2}\right), \\
E_{ \pm \alpha} & =\frac{1}{2}\left(\frac{\lambda_{4}}{2} \pm i \frac{\lambda_{5}}{2}\right), \\
E_{ \pm \beta} & =\frac{1}{2}\left(\frac{\lambda_{6}}{2} \pm i \frac{\lambda_{7}}{2}\right),
\end{aligned}
$$

where roots $(\boldsymbol{\alpha}, \boldsymbol{\beta}, \boldsymbol{\gamma})$ are given as

$$
\begin{aligned}
& \pm \boldsymbol{\alpha}=\left( \pm \frac{1}{2}, \pm \frac{\sqrt{3}}{2}\right), \\
& \pm \boldsymbol{\beta}=\left(\mp \frac{1}{2}, \pm \frac{\sqrt{3}}{2}\right), \\
& \pm \boldsymbol{\gamma}=( \pm 1,0) .
\end{aligned}
$$

Furthermore, we define $h_{\omega}$ :

$$
h_{\omega}=\omega^{i} H_{i}
$$

In order to solve (A3), if we write $\Phi_{3}=\Phi_{3}^{i} H_{i}$, then we have

$$
\left[\Phi_{3}, E_{\boldsymbol{\omega}}\right]=\Phi_{3}^{i} \omega_{i} E_{\boldsymbol{\omega}}
$$

Therefore, when one chooses $\Phi_{3}^{i}$ appropriately, $\Phi_{3}$ commutes with $E_{\boldsymbol{\omega}}$ for a root $\boldsymbol{\omega}$. We choose $\Phi_{3}^{i}$ as to commute with $E_{\gamma}$ here. Then, all generators commuting with $\Phi_{3}$ are $E_{\gamma}, E_{-\gamma}, h_{\gamma}, h$, where $h$ satisfies $\operatorname{tr}\left(h_{\gamma} h\right)=0$ and $\operatorname{tr}(h h)=1 / 2$. Therefore, if $A_{\mu, z}$ are written as the linear combination of those generators, (A3) is satisfied. This is the gauge field on the subgroup $\mathrm{SU}(2) \times \mathrm{U}(1)$, we can written as

$$
A_{\mu, z}=A_{\mu, z}^{1} \frac{\lambda_{1}}{2}+A_{\mu, z}^{2} \frac{\lambda_{2}}{2}+A_{\mu, z}^{3} \frac{\lambda_{3}}{2}+A_{\mu, z}^{8} \frac{\lambda_{8}}{2}
$$

Next, to solve (A1) and (A2), we choose $\Phi_{3}^{i}$ such that we have

$$
\left[\Phi_{3}, E_{\alpha}\right]=\Phi_{3}^{i} \alpha_{i} E_{\alpha}=-E_{\alpha} .
$$

Due to $\boldsymbol{\beta}=\boldsymbol{\alpha}-\boldsymbol{\gamma}$, if we write the form,

$$
\begin{aligned}
& \Phi=\phi_{1} E_{\alpha}+\phi_{2} E_{\beta}, \\
& \tilde{\Phi}=\tilde{\phi}_{1} E_{-\alpha}+\tilde{\phi}_{2} E_{-\beta},
\end{aligned}
$$

(A1) and (A2) are satisfied, where since $\Phi_{1,2}$ is Hermitian matrices, we can show $\tilde{\phi}_{1}=\phi_{1}^{*}$ and $\tilde{\phi}_{2}=\phi_{2}^{*}$.
[1] T. Sakai and S. Sugimoto, Low energy hadron physics in holographic QCD, Prog. Theor. Phys. 113, 843 (2005).

[2] T. Sakai and S. Sugimoto, More on a holographic dual of QCD, Prog. Theor. Phys. 114, 1083 (2005).

[3] Y. Yamaguchi, A. Hosaka, S. Takeuchi, and M. Takizawa, Heavy hadronic molecules with pion exchange and quark core couplings: a guide for practitioners, J. Phys. G 47, 053001 (2020).

[4] A. Hosaka, T. Iijima, K. Miyabayashi, Y. Sakai, and S. Yasui, Exotic hadrons with heavy flavors: X, Y, Z, and related states, Prog. Theor. Exp. Phys. $2016062 \mathrm{C} 01$ (2016).
[5] H.-X. Chen, W. Chen, X. Liu, and S.-L. Zhu, The hiddencharm pentaquark and tetraquark states, Phys. Rep. 639, 1 (2016).

[6] M. Tanabashi et al., Review of particle physics, Phys. Rev. D 98, 030001 (2018).

[7] P. Forgács and N. S. Manton, Space-time symmetries in gauge theories, Commun. Math. Phys. 72, 15 (1980).

[8] N.S. Manton, A new six-dimensional approach to the Weinberg-Salam model, Nucl. Phys. B158, 141 (1979).

[9] C. G. Callan and I. Klebanov, Bound-state approach to strangeness in the Skyrme model, Nucl. Phys. B262, 365 (1985). 
[10] E. Witten, Baryons and branes in anti de Sitter space, J. High Energy Phys. 07 (1998) 006.

[11] M. R. Douglas, Branes within branes, NATO Sci. Ser. C 520, 267 (1999).

[12] H. Hata, T. Sakai, S. Sugimoto, and S. Yamato, Baryons from instantons in holographic QCD, Prog. Theor. Phys. 117, 1157 (2007).

[13] H. Hata and M. Murata, Baryons and the Chern-Simons term in holographic QCD with three flavors, Prog. Theor. Phys. 119, 461 (2008).

[14] The Chern-Simons term is defined properly in an odd dimensional space-time. Here following Ref. [13] we call the CS term for $\int \operatorname{tr} \mathcal{F}^{3}$.

[15] I. Zahed and G. E. Brown, The Skyrme model, Phys. Rep. 142, 1 (1986).

[16] C. G. Callan, K. Hornbostel, and I. Klebanov, Baryon masses in the bound state approach to strangeness in the skyrme model, Phys. Lett. B 202, 269 (1988).

[17] N. S. Manton and P. Sutcliffe, Topological Solitons (Cambridge University Press, Cambridge, England, 2004).
[18] A. V. Manohar and M. B. Wise, Heavy Quark Physics (Cambridge University Press, Cambridge, England, 2000).

[19] C. A. Dominguez and N. Paver, Leptonic decay constants of charm and beauty mesons in QCD, Phys. Lett. B 197, 423 (1987).

[20] Y. Liu and I. Zahed, Heavy baryons and their exotics from instantons in holographic QCD, Phys. Rev. D 95, 116012 (2017).

[21] M. Takayama, H. Toki, and A. Hosaka, Systematics of the SU(3) Baryon Spectra and Deformed Oscillator Quark Model, Prog. Theor. Phys. 101, 1271 (1999).

[22] R. Aaij et al., Observation of $J / \psi p$ Resonances Consistent with Pentaquark States in $\Lambda_{b}^{0} \rightarrow J / \psi K^{-} p$ Decays, Phys. Rev. Lett. 115, 072001 (2015).

[23] R. Aaij et al., Observation of a Narrow Pentaquark State, $P_{c}(4312)^{+}$, and of Two-Peak Structure of the $P_{c}(4450)^{+}$, Phys. Rev. Lett. 122, 222001 (2019). 